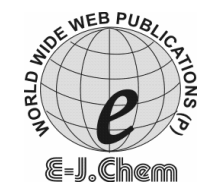

http://www.e-journals.net
ISSN: 0973-4945; CODEN ECJHAO

E-Journal of Chemistry

2009, 6(S1), S304-S310

\title{
Mixed Surfactant Template Method for Preparation of Nanometer Selenium
}

\author{
ZHI-LIN LI and PENG-MIN HUA \\ College of Chemistry and Environmental Science, \\ Hebei University, Baoding-071002, \\ People's Republic of China.
}

Received 1 November 2008; Revised 6 January 2009; Accepted 20 March 2009

\begin{abstract}
Selenium nanoparticles have been synthesized in an aqueous solution by using sodium dodecyl sulfate and polyvinyl alcohol as a soft template. The factors on synthesis, such as reaction time, concentration of reactants and ultrasonic irradiation were studied. The uniform stable selenium nanospheres were obstained in the conditions of 1.0 (mass fraction) sodium dodecyl sulfate, 1.0 (mass fraction) polyvinyl alcohol, $\mathrm{n}(\mathrm{Vc}): \mathrm{n}\left(\mathrm{H}_{2} \mathrm{SeO}_{3}\right)=7: 1$ and 7 minutes after the initiation of the reaction at room temperature. The average particle size of selenium is about $30 \mathrm{~nm}$. The product was characterized by UV and TEM. Finally the applications of the red element nanometer selenium in anti-older cosmetics are presented.
\end{abstract}

Keywords: Selenium nanoparticle, Preparation, Soft template.

\section{Introduction}

Selenium is a trace element essential to the human body. It plays an important physiological function and causes a wide range of pharmacological effects. The amount of selenium can be added to enhance the body immunity and anti-aging. Sodium lauryl sulfate soluble in water, non-toxic, low price has been widely used in cosmetics and detergents. The polyvinyl alcohol is our commonly used facial mask principal constituent. The cost is inexpensive, non-toxic harmless. It has the widespread application in the cosmetics. This experiment discusses the above three materials compound without separation to achieve the goal of nanometer selenium used in the cosmetics ${ }^{1-3}$. In this experiment, nanometer selenium is coated and absorbed by lauryl sodium sulfate and polyvinyl alcohol and the compound state has been good. Moreover it lays aside two months not to precipitate the separation, the stable performance is good.

\section{Experimental}

Sodium lauryl sulfate and polyvinyl alcohol were in aqueous solution with a better ability to dissolve and there is interaction between them ${ }^{4-6}$. Sodium lauryl sulfate can have some influence 
on polymer molecular patterns, thereby changing the nature of the flow of the solution. To a certain concentration it can increase the viscosity of the polymer, and the constitutes micro-environment has a good suspension, emulsion and a stabilizing effect. In the nanometer selenium's preparation response, the precursor $\mathrm{H}_{2} \mathrm{SeO}_{3}$ disperses in the micro environment which in sodium lauryl sulfate and polyvinyl alcohol form, afterward joins antiscorbutic acid and selenious acid has the redox reaction,

$$
\mathrm{H}_{2} \mathrm{SeO}_{3}+2 \mathrm{C}_{6} \mathrm{H}_{6} \mathrm{O}_{6} \rightarrow \mathrm{Se} \downarrow+2 \mathrm{C}_{6} \mathrm{H}_{6} \mathrm{O}_{6}+3 \mathrm{H}_{2} \mathrm{O}
$$

Generated by a single-quality selenium which is coated and absorbed by lauryl sodium sulfate and polyvinyl alcohol, so as to effectively prevent the initial formation of selenium polymer particles, mutual polymerization and reunion and slow and control the growth of selenium particles. From the experimental condition is obtained nanometer selenium for red or orange, and refers to generate a single quality of the existence of selenium in the form of amorphous or single-crystal state ${ }^{7}$.

A certain amount of selenious acid, lauryl sodium sulfate solution and polyethylene alcoholic solution was taken in a $15 \mathrm{~mL}$ test tube, evenly mixed and then ascorbic acid solution was added, was vigorously shaked, after the mix, settle period of time, Se returns to single-quality selenium. A different reaction condition of the reaction solution was taken in deionized water to make up a reference in the $450 \mathrm{~nm}$ and $520 \mathrm{~nm}$ wavelengths measure the absorption solution response, and the calculation of the two wavelengths of the absorption ratio. And then do a product on the morphology of TEM observation and analysis.

\section{Results and Discussion}

\section{Absorption spectrometry}

Figure 1 is the different solution of the absorption curve. As can be seen from Figure 1, ascorbic acid, $\mathrm{H}_{2} \mathrm{SeO}_{3}$, sodium lauryl sulfate and polyvinyl alcohol absorb not obviously in the visible light area, but the reacting system $\left(0.01 \mathrm{~mol} / \mathrm{L} \mathrm{H}_{2} \mathrm{SeO}_{3}\right.$ solve in the mass fraction of $1.0 \%$ sodium lauryl sulfate and polyvinyl alcohol react with $0.07 \mathrm{~mol} / \mathrm{L} \mathrm{Vc}$ ) the absorption is big, it is explained that the respond component may neglect the reacting system absorption's influence.

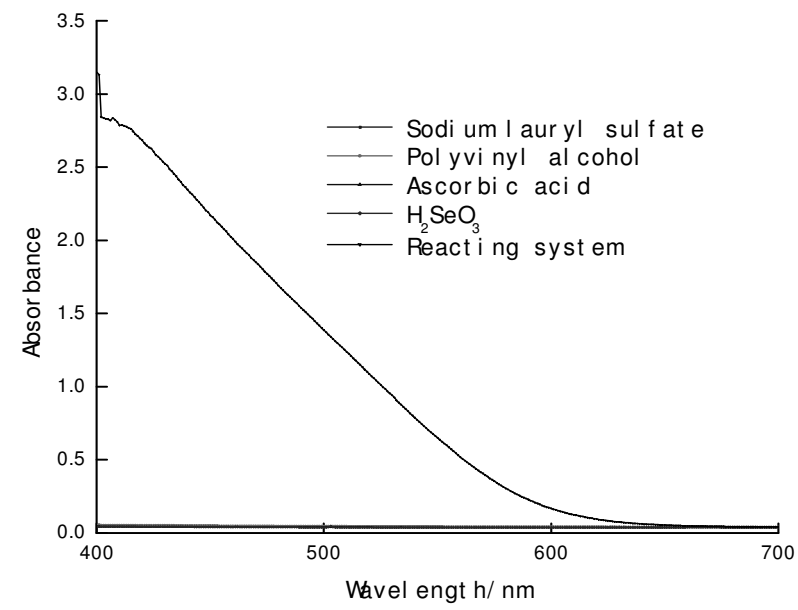

Figure 1. Absorption spectra of Vc solution; $\mathrm{H}_{2} \mathrm{SeO}_{3}$; Sodium lauryl sulfate; Polyvinyl alcohol. 


\section{Effect of contact time}

According to the determination of colloidal solution of the two-wavelength method, the colloidal particle size parameters $B=\lg \left(A_{2} / A_{1}\right) / \lg \left(\lambda_{1} / \lambda_{2}\right)$, of which $A_{2}$ and $A_{1}$ were wavelength $\lambda_{2}$ and $\lambda_{1}$ under the absorbance value. From this we can see and fix wavelength $\lambda_{1}$ and $\lambda_{2}$, if the ratio of absorbance $A_{2} / A_{1}$ remain unchanged when the colloidal particle size is in a steady state. In order to facilitate the determination of this paper to choose district Vis $450 \mathrm{~nm}$ and $520 \mathrm{~nm}$ as the nano-selenium solution's determination wavelength, and use the two wavelength absorption ratio as the characterization of product size over time based on. Figure 2 is a response to a fixed system $\mathrm{H}_{2} \mathrm{SeO}_{3}$ concentration of $0.01 \mathrm{~mol} / \mathrm{L}, \mathrm{Vc}$ concentration of $0.07 \mathrm{~mol} / \mathrm{L}$, sodium lauryl sulfate mass fraction of 1.0 percent, the mass fraction of polyvinyl alcohol for 1.0 percent, at $25{ }^{\circ} \mathrm{C}$ when the absorbance with time curve.

As the time goes the absorption of the solution was increased as shown in Figure 2. After 7 minutes the absorption of the solution dose not change, it shows that it has achieved a balanced response, and the two wavelengths of the absorption ratio remained at around 2, stabilized at this time that the formation of nano-particles of selenium in a uniform steady state.

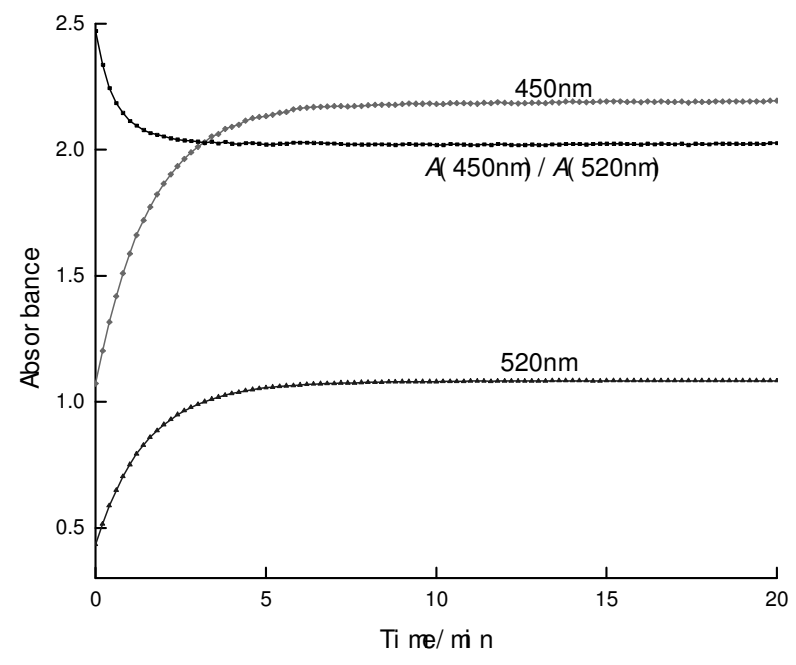

Figure 2. Plots of reaction time $v s$. absorbance of product.

\section{Effect of ascorbic acid}

Figure 3 is when selenious acid concentration of $0.01 \mathrm{~mol} / \mathrm{L}$, sodium lauryl sulfate mass fraction of 1.0 percent and the mass fraction of polyvinyl alcohol for 1.0 percent, to change the concentration of ascorbic acid, and then to acquire the reaction solution absorption curve (When $25{ }^{\circ} \mathrm{C}$ responds 7 minutes $)^{8}$. As shown in Figure 3, with the increase in the amount of ascorbic acid, the solution absorbance under the two wave lengths is gradually increasing, then gradually reduced and stabilized, indicating that the response tends completely. In $10 \mathrm{~mL}$ response solution, when the concentration of ascorbic acid is $0.07 \mathrm{~mol} / \mathrm{L}$, the solution absorption ratio stabilizes basically about 2 to illustrate that at this point the nano-Se solution was uniform stability. 


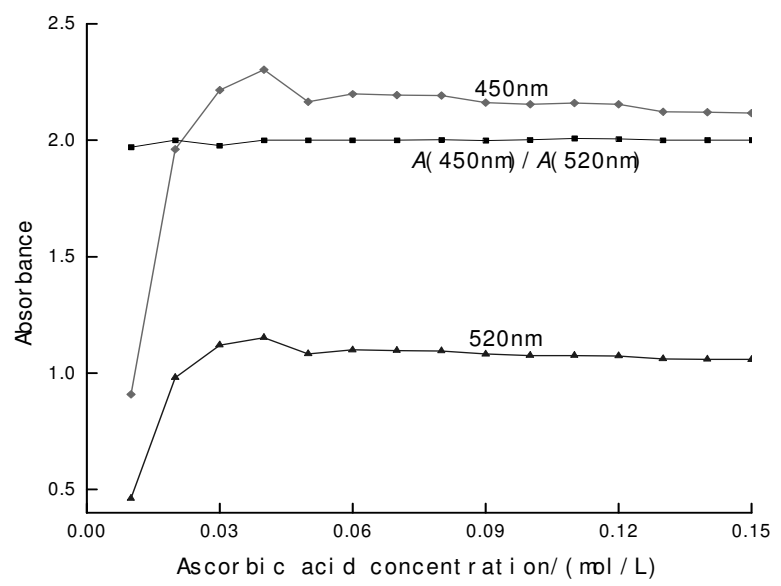

Figure 3. Plots of Vc concentration $v s$. absorbance of product

\section{Effect of sodium lauryl sulfate}

Figure 4 is when selenious acid concentration of $0.01 \mathrm{~mol} / \mathrm{L}$, ascorbic acid concentration of $0.07 \mathrm{~mol} / \mathrm{L}$ and the mass fraction of polyvinyl alcohol for 1.0 percent, to change the amount of sodium lauryl sulfate, then to acquire the reaction solution absorption curve(When $25{ }^{\circ} \mathrm{C}$ responds 7 minutes) $)^{9}$. As presented in Figure 4, with the increase in the amount of sodium lauryl sulfate, the solution absorbance under the two wave lengths is gradually decreasing. When the mass fraction of sodium lauryl sulfate is 0.6 percent, the solution absorption ratio stabilizes basically about 2 to illustrate that at this point the nano-Se solution uniform stability.

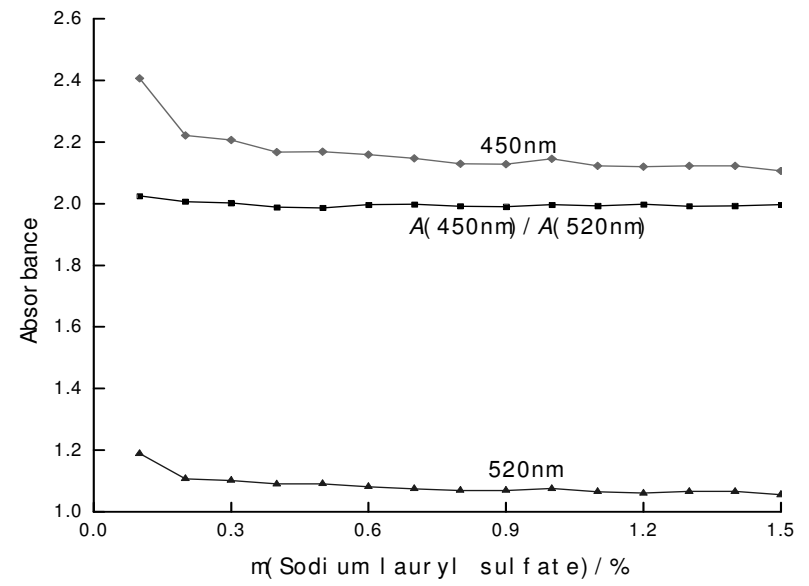

Figure 4. Plots of sodium dodecyl sulfate mass fraction $v s$. absorbance of product

\section{Effect of polyvinyl alcohol}

Figure 5 is when selenious acid concentration of $0.01 \mathrm{~mol} / \mathrm{L}$, ascorbic acid concentration of $0.07 \mathrm{~mol} / \mathrm{L}$ and sodium lauryl sulfate mass fraction of 1.0 percent, to change the amount of polyvinyl alcohol, then to acquire the reaction solution absorption curve (When $25^{\circ} \mathrm{C}$ responds 7 minutes). As shown in Figure 5, with the increase in the amount of polyvinyl alcohol, the solution absorbance under the two wave lengths is gradually decreasing. When the mass 
fraction of polyvinyl alcohol is 0.6 percent, the solution absorption ratio stabilizes basically about 2 to illustrate that at this point the nano-Se solution uniform stability.

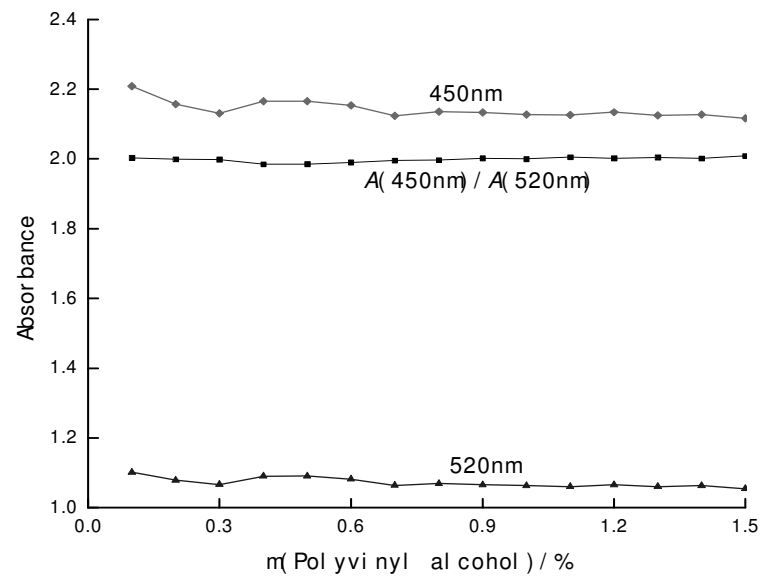

Figure 5. Plots of polyvinyl alcohol mass fraction $v s$. absorbance of product

In the experiment when the reaction system without a template for sodium lauryl sulfate and polyvinyl alcohol, in a test tube by adding sub-ascorbic acid and selenious acid, the colorless test tube solution generated by the light yellow, along with time increase, the color deepens gradually, moreover presents a muddy, this explained that the response production the simple substance selenium (Se) could not exist stably, occur in the coagulation, and the test-tube has the red sediment formation.

\section{Effect of selenious acid}

Figure 6 is when ascorbic acid concentration of $0.07 \mathrm{~mol} / \mathrm{L}$, sodium lauryl sulfate mass fraction of 1.0 percent and the mass fraction of polyvinyl alcohol for 1.0 percent, to change the concentration of selenious acid, and then to acquire the reaction solution absorption curve (When $25^{\circ} \mathrm{C}$ responds 7 minutes). As shown in Figure 6, with the increase in the amount of selenious acid, the solution absorbance under the two wave lengths is increasing. When the concentration of the selenious acid is $0.01 \mathrm{~mol} / \mathrm{L}$, the solution absorption ratio stabilizes basically about 2 to illustrate that at this point the nano-Se solution uniform stability.

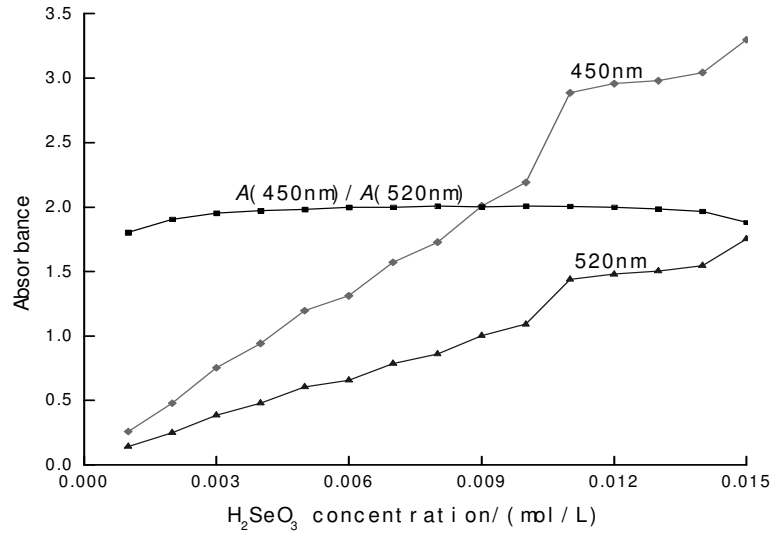

Figure 6. Plots of $\mathrm{H}_{2} \mathrm{SeO}_{3}$ concentration vs. absorbance of product. 


\section{Morphology characteristics of the product under the TEM}

From the TEM photographs can be seen, this reacting system forms a nanometer selenium is the spherical particle, and evenly dispersed, with an average diameter of about $30 \mathrm{~nm}$ and is able to exist stably of a month or so. At normal temperature let the ultrasonic cleaning device deal with the reacting system for an hour, the solution can be stored for more than two months. Observing under the lens, the particle dispersion is good.

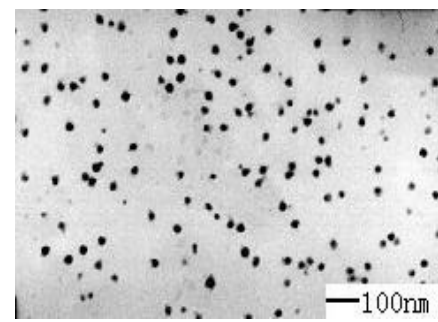

Figure 7. Not supersonic

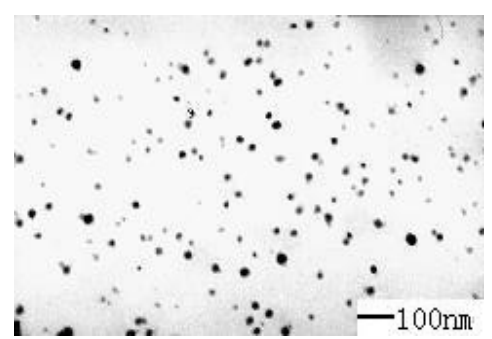

Figure 8. Supersonic 1 hour

The application of nanometer selenium in cosmetics

The preparation of selenium cosmetics.

\begin{tabular}{lcc}
\hline \multicolumn{1}{c}{ Raw material name } & Content, w/\% & Note \\
\hline Glycerol & 10.0 & $\mathrm{~A}$ \\
Allantoin & 0.2 & \\
Surface active agent A & 0.3 & \\
Surface active agent B & 0.6 & \\
Antiseptic A & 0.1 & \\
nanometer selenium & 0.03 & \\
$\mathrm{H}_{2} \mathrm{O}$ & 69.0 & \\
White oil & 6.2 & $\mathrm{~B}$ \\
Silicone oil & 2.0 & \\
Octadecyl alcohol & 8.2 & \\
Single glyceride & 1.6 & \\
Beeswax & 1.6 & \\
Antiseptic B & 0.1 & \\
Essence & 0.1 & $\mathrm{C}$ \\
\hline
\end{tabular}

\section{Preparation}

1. Heat up A component and B component separately to $95^{\circ} \mathrm{C}$ and maintain this temperature 20 minutes.

2. Under the agitation joins the B component in A component, the temperature control in $85 \pm 5^{\circ} \mathrm{C}$, stirs 20 minutes.

3. When the temperature drop to $45^{\circ} \mathrm{C}$ joins the essence, continues to stir.

4. When the temperature drop to $40{ }^{\circ} \mathrm{C}$ then stops the agitation and obtains the product.

\section{Selenium cosmetics technical performance determination}

The product sensory index, the physics and chemistry target, and the hygienic target have been test refer to the People's Republic of China light industry profession related standard and conforms to the national standards. 


\section{Conclusion}

This experiment with mixed template synthesis of nano-selenium, through to experimental condition's discussion and the fumble, has made the satisfactory results. Moreover sodium lauryl sulfate and polyvinyl alcohol the prices are low, the cost is inexpensive, non-toxic and does not have the side effect. Let the ultrasonic cleaning device deal with the product, it can be stored for more than two months. The nanometer red element selenium can eliminate the free radical effectively, postpone senility. We act according to the skin senile principle, uses the scientific formula, matches again by the moderate chemical additive and prepares the nanometer selenium facial cream. After examines its sensory index, physics and chemistry target, hygienic target to be able to meet the related standard requirements, it is one kind of good functionality anti-senile cosmetics product, and has the good development value and the development prospect.

\section{References}

1. Abdelouas A, Gong W L and Lutze W, Shelnutt J A, Franco R and Moura I, Chem Mater., 2000, 12, 1510.

2. Yu Xiafei and Gao Xueyun, Nanometer selenium on medicine filter material application, China high technology and new technology Enterprise, 2000, 6, 33-34.

3. Gao X Y, Zhang J S and Zhang L D, Adv Mater., 2002, 14(4), 290-293.

5. Wang Hongyan, Zhang Shengyi, Hu Hong Wen, SHU Zi-Ying, FU Gui-Xiang, BO Jia-Lai, CHENG Fu-Yu and HU Yu-Jie, Functional Materials, 2004, 35(2), 259-261.

6. Wang Hongyan, Zhang Li, Zhang Shengyi, Yunyang Dai, Qing Li, Qi Zhang and Jay Kuo C C, J Huaibei Coal Industry Teachers College, 2004, 25(3), 36-39.

7. Shi Hongwei and Song Jiming, Journal of Jiaying University (Natural Sciences), 2004, 22(3), 27-30.

8. Gates B, Mayers B Cattle B and Xia Y, Adv Funct Mater., 2002, 12(3), 219-227.

9. David R M, William P and Peter J Tarcha, J Colloid and Interface Science, 1995, 170, 254-260. 


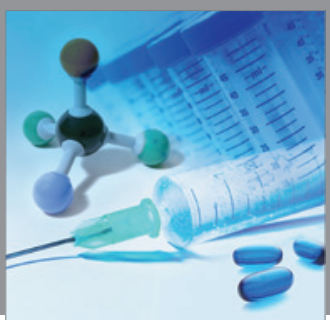

International Journal of

Medicinal Chemistry

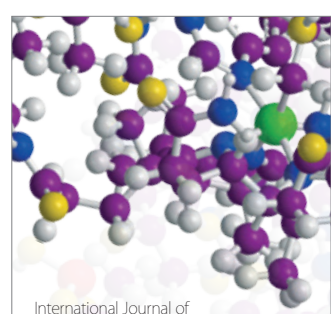

Carbohydrate Chemistry

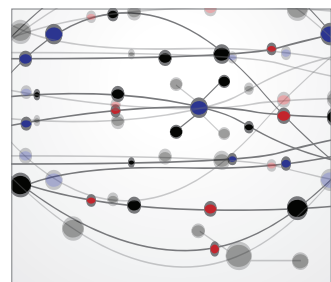

The Scientific World Journal
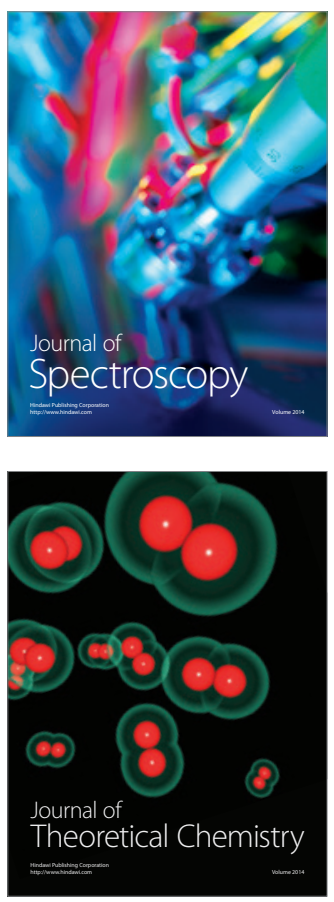
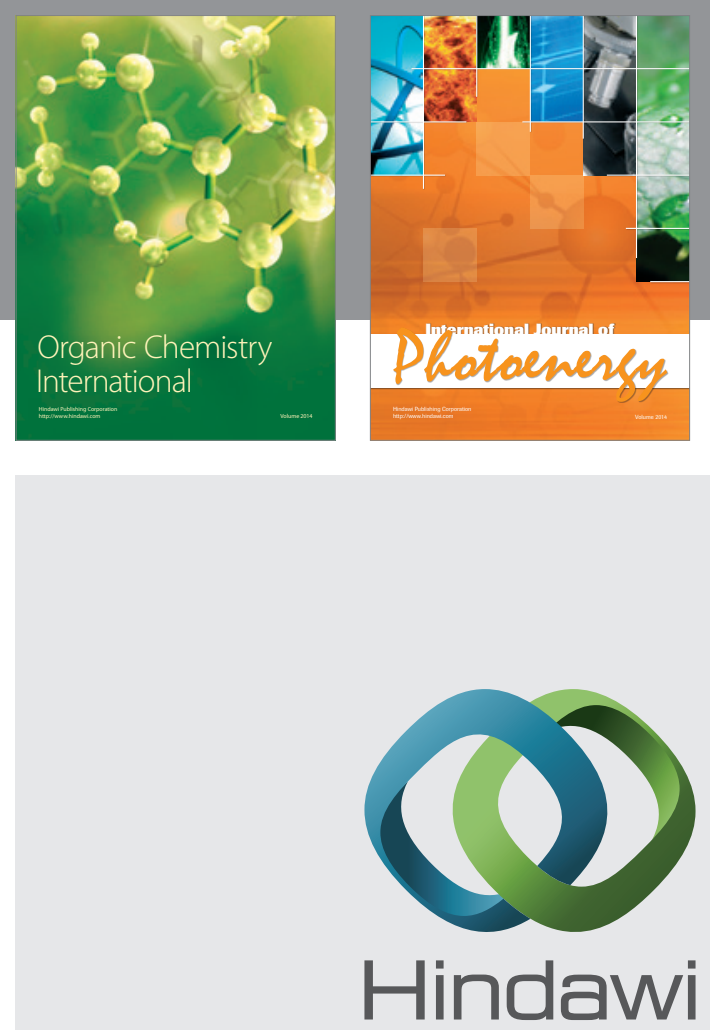

Submit your manuscripts at

http://www.hindawi.com
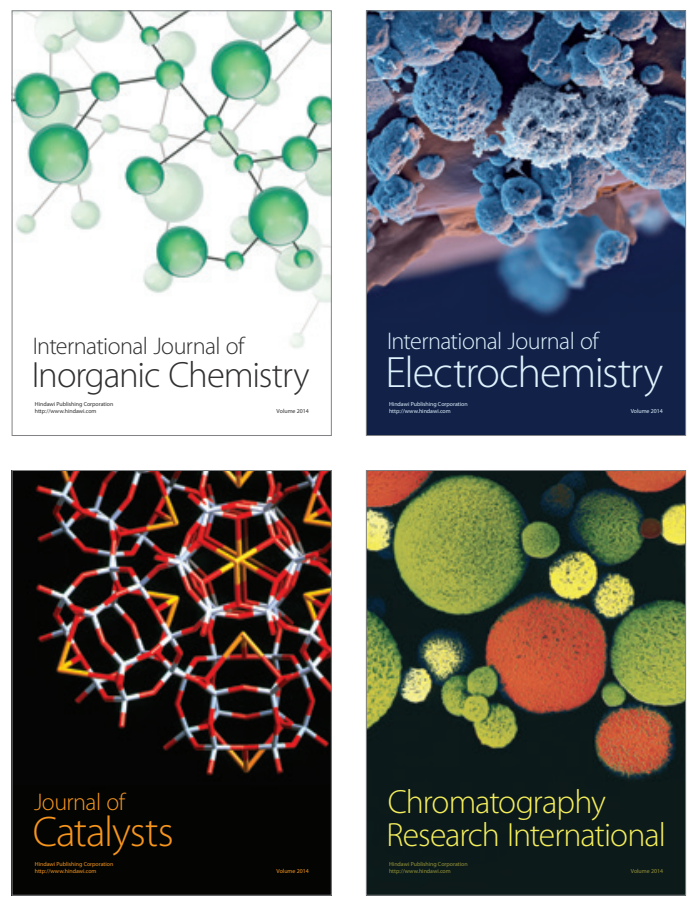
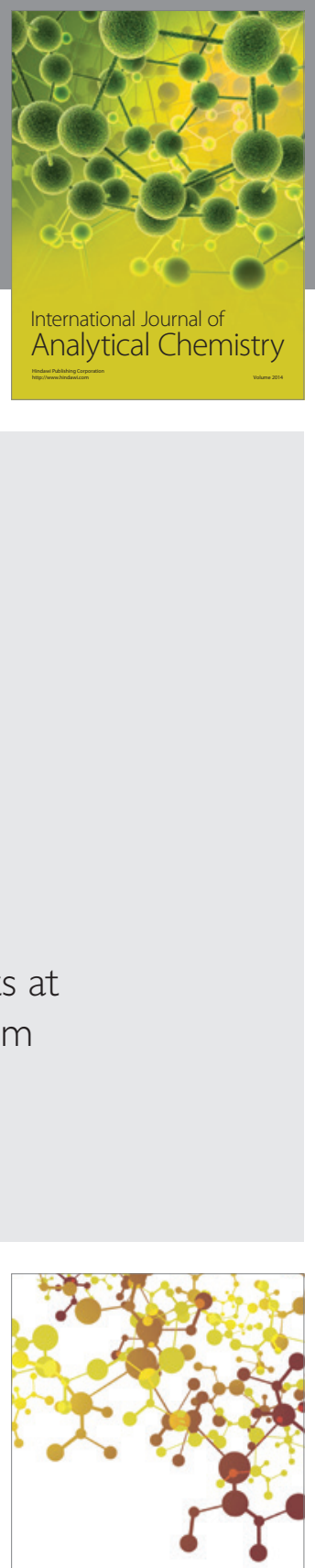

Journal of

Applied Chemistry
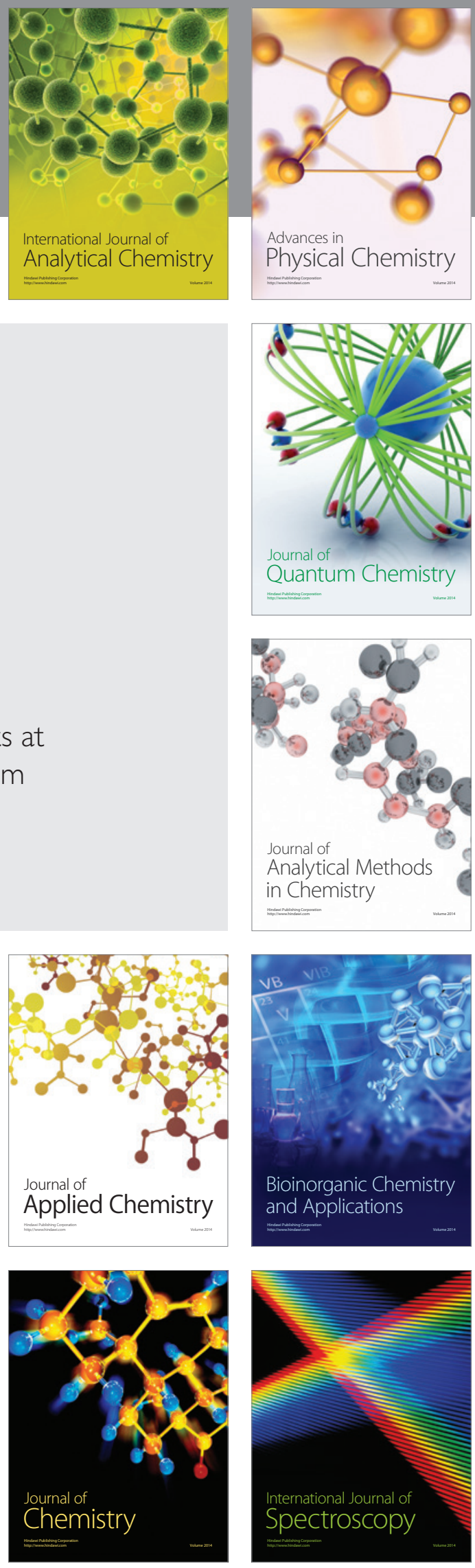